\section{Cross-modal reversal in the rabbit}

\author{
JEANETTE P. WARD and ARTHLR L. Y'EHLE \\ Memphis State Linversity. Memphus. Tenn. 38111
}

A classically conditioned discrimination was established between two rates of intermittent stimulation in the auditory modality for four rabbits and in the visual modality for four additional rabbits. Following acquisition in the original modality, both stimulus modality and stimulus contingencies were changed in a cross-modal reversal test. All rabbits were subsequently tested in reacquisition of the original discrimination. Results were compared to a previous test of direct cross-modal transfer. It was concluded that results of cross-modal reversal support the earlier finding of cross-modal transfer in the rabbit.

In a recent study (Yehle \& Ward, 1969), it was found that rabbits classically conditioned to discriminate between two rates of intermittent stimuli in ejther the visual or the auditory modality were able to maintain the discrimination of intermittent rate when sense modality was changed. Although the facilitation of performance in the transfer condition strongly indicated transfer of the specific discrimination across sense modalities, the extremely rapid learning that is often observed with this type of measure made interpretation of the results somewhat difficult.

In a subsequent study of cross-modal transfer in the bushbaby, an experimental design was used which proved much more satisfactory in the clear delineation of transfer independent of the effects of rate of learning (Ward, Yehle. \& Doerflein, in press). In this study some animals were given a direct transfer, i.e., stimulus contingencies were maintained the same across sense modalities, while other animals were given a reversal transfer, i.e., stimulus contingencies were reversed when sense modality was changed. This type of design has been used previously both with positive (Wilson \& Shaffer. 1963) and with nega tive (Wegener, 1965: Ettlinger \& Blakemore, 1966) results.

Since the initial study of transfer in the rabbit used only the direct transfer paradigm, it seemed desirable to test the strength of the transfer effect with a reversal test. In this study rabbits were trained to make a classically conditioned discrimination of intermittent visual or auditory stimuli and, subsequently. were tested with the stimulus contingency reversed in the second modality.

\section{METHOD}

The Ss were eight New Zealand albino rabbits, approximately 14 weeks old and weighing between 5 and $5 \% \frac{1}{2}$ pounds. Each $S$ was caged individually and had free access to food and water.

During training the Ss were restrained in a Plexiglas box that was placed in a dark ventilated sound-attenuated chamber. The eyeblink (EB) response was recorded by the use of a stainless steel suture inserted into the skin of both the upper and lower eyelid of the $S^{\prime}$ 's right eye. Wireform connectors clamped to the free ends of the suture were connected to a Grass polygraph. (This response measure is similar to that discussed by Vandercar, Swadlow. Elster, \& Schneiderman, 1969.) Two stainless steel safety pins were inserted chronically into the skin of each S to record the heart rate (HR).

The CSs were either $700-\mathrm{Hz}$ tone pulses presented to the $S$ through a $6 \cdot$ in. speaker, located 4 in. above his head, or light flashes from a $28 . \mathrm{V}$ incandescent bulb located similarly. The US was an ac electric shock of $.3 \cdot \mathrm{sec}$ duration, administered through the stainless steel eyelid suture described above. In order to maintain a stable level of HR response. shock intensity was varied from 2 to $12 \mathrm{~mA}$ over days of training. The CS duration was $2.3 \mathrm{sec}$. and the offset of the CS was coincident with the offset of the US on CS+ trials. The intertrial interval was $69 \mathrm{sec}$.

Initial discrimination acquisition was with auditory stimuli for the first group of four $\mathrm{Ss}$ and with visual stimuli for the second group of four Ss. For both the auditory and visual conditions, CS+ was composed of intermittent pulses at the rate of two per second, with a duration of $20 \mathrm{msec}$, and CS - at the rate of eight per second, with a duration of $40 \mathrm{msec}$.

Following 13 days of initial discrimination acquisition, the $C S$ contingencies were reversed $(\mathrm{CS}+=$ eight per second and $\mathrm{CS}-=$ two per second) and the modalities of stimulation were changed, from auditory to visual for the first group and from visual to auditory for the second group.

Upon completion of 13 days of reversal training in the second modality, all Ss were run for a final 13 days under the original conditions of initial discrimination acquisition.

During each daily session, Ss received 24 trials with the CSt and 24 trials with the CS -, presented randomly with the

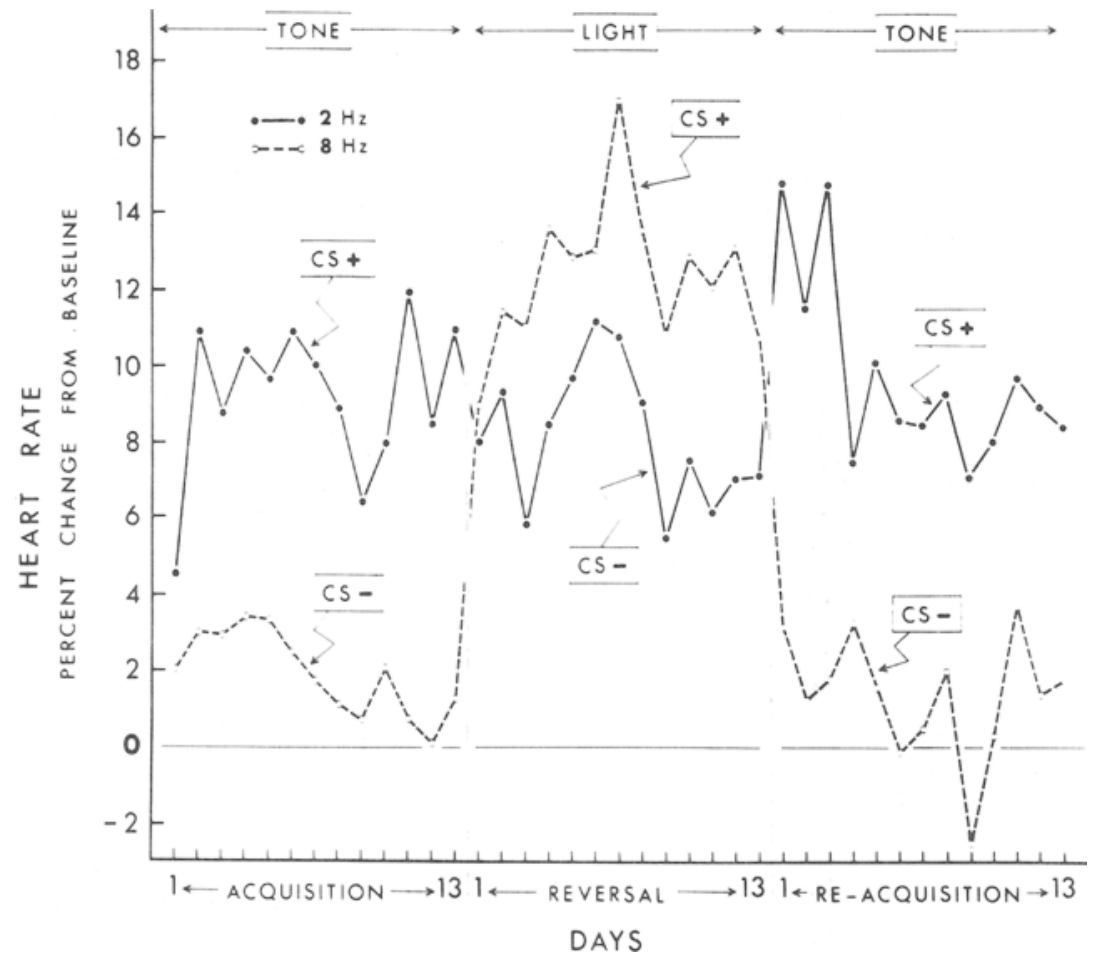

Fig. 1. Mean HR responses to CS+ and $\mathrm{CS}-$ in acquisition of an auditory discrimination, reversal of stimulus contingencies in the visual modality, and reacquisition of the original auditory discrimination. 


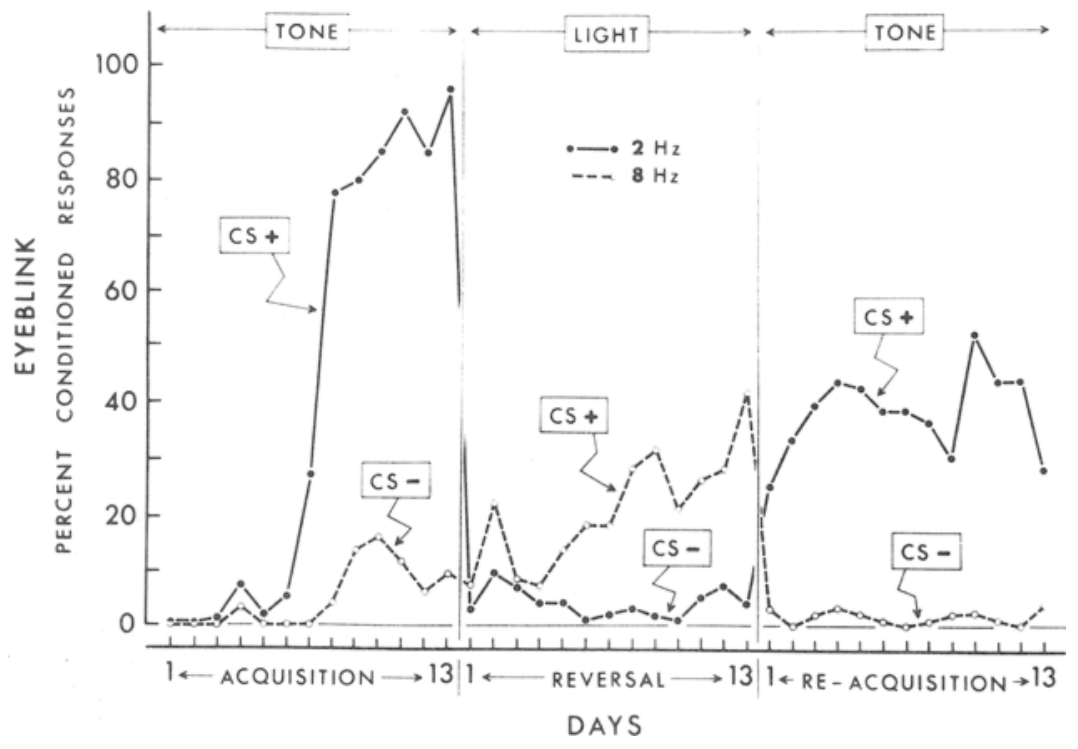

Fig. 2. Mean EB responses to CS+ and CS- in acquisition of an auditory discrimination, reversal of stimulus contingencies in the visual modality, and reacquisition of the original auditory discrimination.

restriction of no more than two similar and a $5-\mathrm{mm}$ pen deflection corresponding trials in succession. Trials 11 and 12,23 and 24,35 and 36 , and 47 and 48 were designated as test trials (no US) and were used to assess $\mathrm{HR}$ responding to the CS+ and the CS- by measuring the distance between 10 successive heart beats prior to CS onset and comparing this with the measurement of 10 successive heart beats immediately following CS onset. A percent change from baseline was then calculated and used as a measure of HR CRs. The EB responding was measured on every trial,

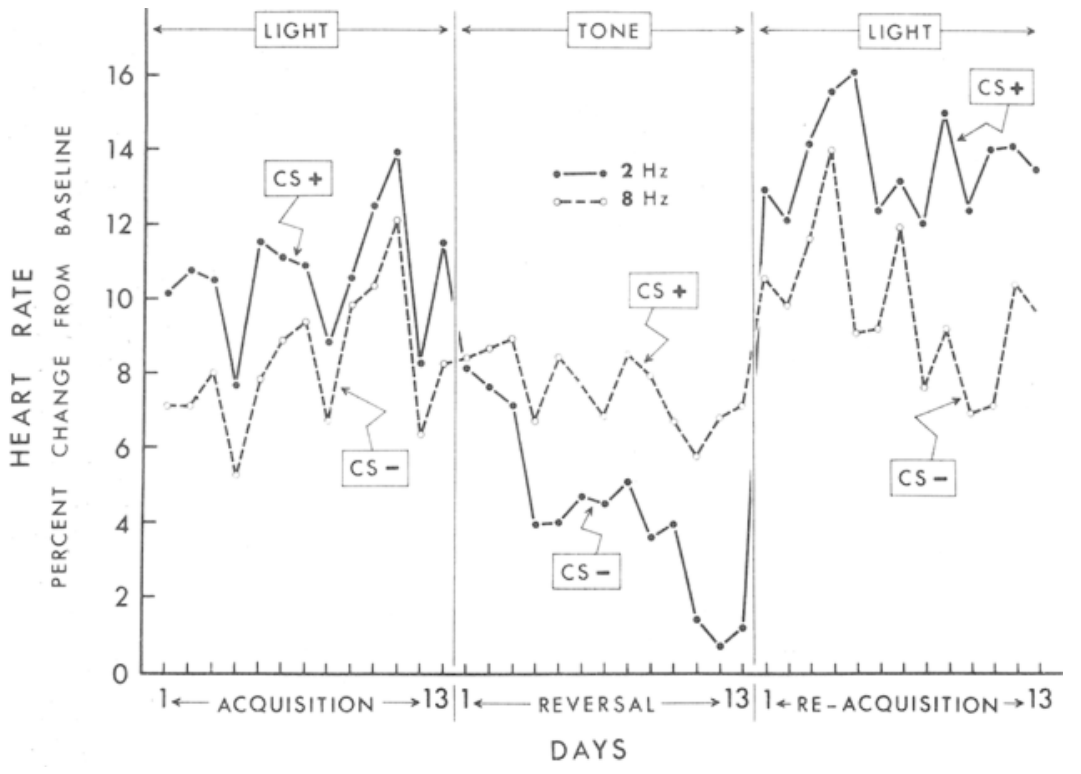

Fig. 3. Mean $\mathrm{HR}$ responses to $\mathrm{CS}+$ and $\mathrm{CS}-$ in acquisition of a visual discrimination, reversal of stimulus contingencies in the auditory modality and reacquisition of the original visual discrimination. appear until Session 7 or 8 . The magnitude of the discrimination as measured by the difference in responding to the $\mathrm{CS}+$ and CS was greater to the auditory than to the visual stimuli for both the HR and EB responses. Both phenomena, the more rapid manifestation of learning by the $H R$ response and also the superior effectiveness of the auditory stimuli for the rabbit in this test, were observed previously in the direct transfer experiment (Yehle \& Ward, 1969).

Examination of Figs. 1 and 3 shows that the magnitude of the HR discrimination was smaller in the first sessions of the reversal transfer condition than in the first sessions of initial acquisition. This fact suggests that the performance of the rabbits was adversely affected in the transfer condition by the reversal of stimulus contingencies. However, as in the previous direct transfer study, the very rapid acquisition of this classically conditioned discrimination makes conclusions about transfer based on rate of learning difficult. Comparison of discrimination performance in the reversal transfer condition in this study with the performance of Ss in direct transfer in the previous study (Yehle \& Ward, 1969) constitutes a measure not dependent on learning rates (Table 1). A paired comparison of mean difference scores from these two studies indicates that $\mathrm{Ss}$ which had reversal of stimulus contingencies in the second modality gave less evidence of discrimination in the first session of transfer than did Ss which had a direct transfer of stimulus contingencies to the new modality $(t=5.08, \quad d f=3$, $\mathrm{p}<.01)-$ or conversely, that rabbits are indeed facilitated in the discrimination of intermittent stimuli as a consequence of prior experience with the same stimulus contingencies in another modality.

The final test, which was a reacquisition of the initial discrimination, was included in this test series as a measure of the strength of cross-modal transfer. If the intersensory effect was strong, it would be

Table 1

Mean Magnitude* of Discrimination on First Transfer Session for Ss with Direct** and Reversal Conditions

\begin{tabular}{lcc}
\hline & Direct & Reversal \\
\hline EB (Tone) & 9.0 & 2.8 \\
EB (Light) & 6.5 & 1.0 \\
HR (Tone) & 6.5 & 0.3 \\
HR (Light) & 3.8 & 0.9 \\
\hline
\end{tabular}

*Magnitude of discrinination for each $S$ is scored in the $E B$ as the difference in number of $C R s$ and in $H R$ as the difference in percent $C R$ above baseline between $\mathrm{CS}+$ and $\mathrm{CS}_{-}$ responses.

* Data from $S S$ with direct transfer is from a previous study /Ychle \& Ward. 1969). 


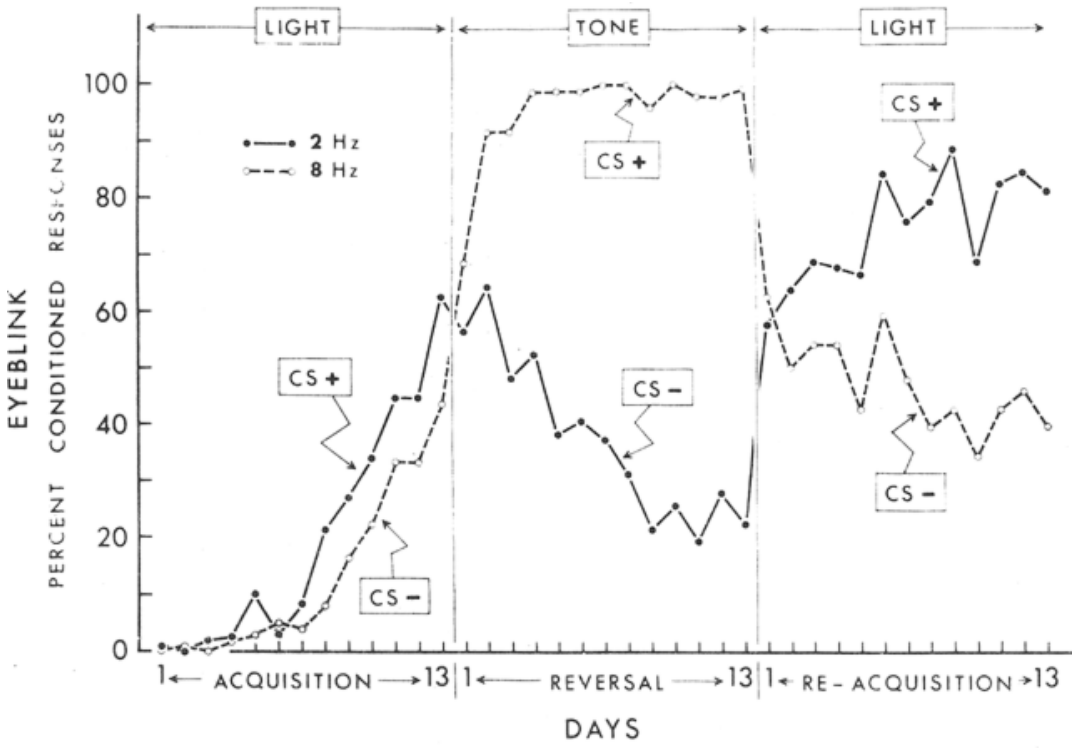

Fig. 4. Mean EB responses to $\mathrm{CS}+$ and $\mathrm{CS}$ - in acquisition of a visual discrimination, reversal of stimulus contingencies in the auditory modality and reacquisition of the original visual discrimination.

expected that reversal in the second modality would result in a deficit in the performance of the initial discrimination. Little evidence for such a deficit was observed in reacquisition. Instead, responding was promptly resumed at the level of initial acquisition.

The cross-modal transfer effect in lower animals is not strong. Evidence of this fact is found in the many reports of negative results in the literature (cf. Burton \& Ettlinger, 1960; Ettlinger, 1960; Wegener, 1965; Rothblat \& Wilson, 1968). Some of the factors related to the difficulties in measuring this phenomenon have been discussed elsewhere (Ward et al, in press). Chief among these is the reliance on comparison of rates of learning. The direct and reversal transfer paradigm, which depends on comparison of performance in the early trials of transfer only, obviates this difficulty. Although cross-modal transfer is not a strong effect, recent reports of transfer in mice (Oliverio \& Bovet, 1969) and in rats (Over \& Mackintosh, 1969), taken togehter with these studies on the rabbit, indicate that the effect is present even in the simpler species.
REFHRENCES

BURTON, D., \& ETTI.INGER, G. Cross-modal transfer of training in monkeys. Nature, 1960 , 186, 1071-1072.

FTTLINGER, G. Cross-model transfer of training in monkeys. Behaviour, 1960, 16, 56-65.

ETTLINGER, G., \& BLAKEMORE, C. B. Cross-model transfer to conditional discrimination training in monkeys. Nature, 1966, 210, 117-118.

OLIVERIO, A., \& BOVET, D. Transfer of avoidance responding between visual and auditive stimuli presented in different temporal patterns. Communications in Behavioral Biology, Part A, 1969, 3, 61-68.

OVER, R., \& MACKINTOSH, N. J. Cross-modal transfer of intensity discrimination by rats. Nature, 1969, 224, 918-919.

ROTHBLAT, L. A., \& WILSON, W. A., JR. Intradimensional and extradimensional shifts in the monkey within and across sensory modalities. Journal of Comparative \& Physiological Psychology, 1968, 66, 549-553.

VANDERCAR, D. H., SWADLOW, H. A., ELSTER, A., \& SCHNEIDERMAN, N. Nictitating membrane with corneo-retinal transducers for conditioning in rabbits. American Psychologist, 1969, 24, 262-264.

WARD, J. P., YEHLE, A. L., \& DOERFLEIN, R. $S$. Cross-modal transfer of a specific discrimination in the bushbaby, Galago senegalensis. Journal of Comparative \& Physiological Psychology, in press.

WEGENER, J. G. Cross-modal transfer in monkeys. Journal of Comparative \& Physiological Psychology, 1965, 59, 450-452. WIISON, W. A., \& SHAFFER, O. C. Intermodality transfer of specific discriminations in the monkey. Nature, 1963, 197, 107.

YEHLE, A. L., \& WARD, J. P. Cross-modal transfer of a specific discrimination in the rabbit. Psychonomic Science, 1969, 16, 269-270. 